\title{
Attenuation of Perfluorooctane Sulfonate-Induced Steatohepatitis by Grape Seed Proanthocyanidin Extract in Mice
}

\author{
Tao Huang $\mathbb{D}^{\mathbb{D}}$, Yurong Zhang $\mathbb{D}^{\mathbb{D}}$, Wenjuan Zhang $\mathbb{D}^{\mathbb{D}}$, Tingting Lin $\mathbb{D}^{\mathrm{D}}$, Luoting Chen $(\mathbb{D}$,

 \\ Department of Physiology, Medical College of Nanchang University, Nanchang 330006, China \\ Correspondence should be addressed to Dalei Zhang; zhangdalei@ncu.edu.cn
}

Received 31 August 2020; Revised 11 November 2020; Accepted 30 November 2020; Published 9 December 2020

Academic Editor: Aleksandra Buha

Copyright (C) 2020 Tao Huang et al. This is an open access article distributed under the Creative Commons Attribution License, which permits unrestricted use, distribution, and reproduction in any medium, provided the original work is properly cited.

\begin{abstract}
Perfluorooctane sulfonate (PFOS), an environmentally persistent pollutant, has been revealed to elicit hepatic toxicity. In the current study, we investigated the protective role of grape seed proanthocyanidin extract (GSPE) against PFOS-caused steatohepatitis in mice. Animals were exposed intragastrically to PFOS (10 mg/kg/day), GSPE ( $150 \mathrm{mg} / \mathrm{kg} / \mathrm{day})$, or their combination. After 21 days of treatment, mice exposed to PFOS exhibited steatosis, oxidative stress, and inflammation in the liver. Nevertheless, simultaneous administration of GSPE resumed the declined serum hepatic enzyme activities and histological abnormalities in PFOS-exposed mice. Furthermore, GSPE supplementation reduced the contents of triglyceride (TG) and total cholesterol (TC) and expression of lipid metabolism-associated genes CD36 and fatty acid-binding protein 4 (FABP4) in the liver of mice treated with PFOS. Moreover, GSPE suppressed the generation of lipid peroxidative product malondialdehyde and restored the activity of superoxide dismutase in the liver of PFOS-exposed mice. In addition, GSPE repressed the PFOS-induced hepatic overproduction of proinflammatory cytokines interleukin-6 (IL-6) and tumor necrosis factor- $\alpha$ (TNF- $\alpha$ ). Our results demonstrate that GSPE attenuates PFOS-caused steatohepatitis in mice by regulating lipid metabolism, oxidative stress, and inflammatory response.
\end{abstract}

\section{Introduction}

Steatohepatitis is a common histological finding that involves a variety of etiologies. Toxicant-associated steatohepatitis is one of the most frequent causes of steatohepatitis [1]. Perfluorooctane sulfonate (PFOS), a member of a family of perfluoroalkyl substances (PFAS), has widely aroused public attention due to its ubiquitous distribution, environmental persistence, high bioaccumulation, and potential toxicity [2]. PFOS has been detected in populations worldwide [3], and the consumption of contaminated foods and drinking water and inhalation of indoor dust are the predominant exposure pathways of PFOS to nonoccupationally exposed people [4]. Owing to the long half-life of elimination in people (approximately 5 years) [5], PFOS accumulates in the body and poses a threat to human health, particularly for occupationally exposed persons. Tests in rodents have revealed that PFOS exposure caused hepatic steatosis [6-9].
In humans, monitoring studies have also reported that serum PFOS is significantly associated with dyslipidemia $[10,11]$. Furthermore, PFAS exposure is correlated with increased risk of steatohepatitis and fibrosis in children diagnosed with nonalcoholic fatty liver disease (NAFLD), suggesting that PFOS may be a toxicant contributing to nonalcoholic steatohepatitis (NASH) [12].

Nutrition is a powerful protector against environmental chemical insults [13]. Proanthocyanidins are a class of polyphenols that possess a wide range of health benefits [14]. Grape seed proanthocyanidin extract (GSPE) is a major source of proanthocyanidins. The physiological activities of proanthocyanidins include reduction in oxidative stress, inflammation, and metabolic syndrome which provide multiorgan protection from various drug- and chemicalinduced toxicities $[15,16]$. Although several studies have shown the protective effect of GSPE on hepatotoxicity induced by diverse environmental pollutants [17-21], it 


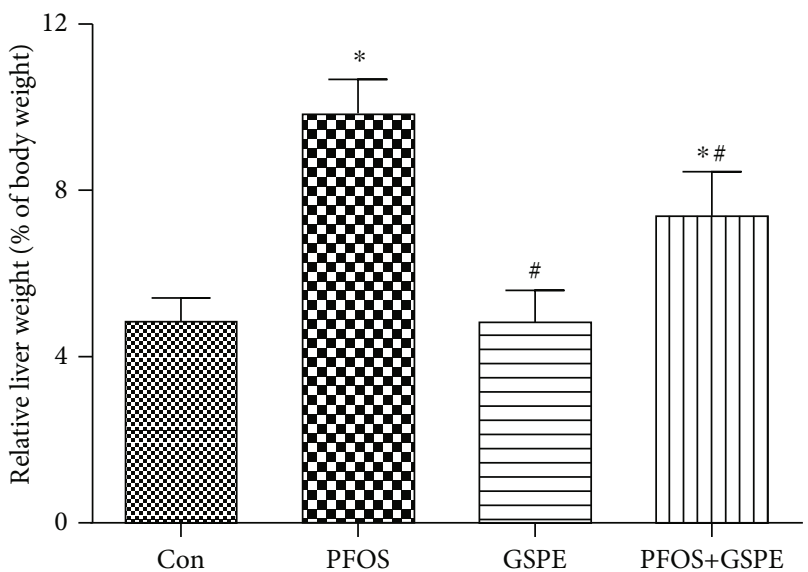

(a)

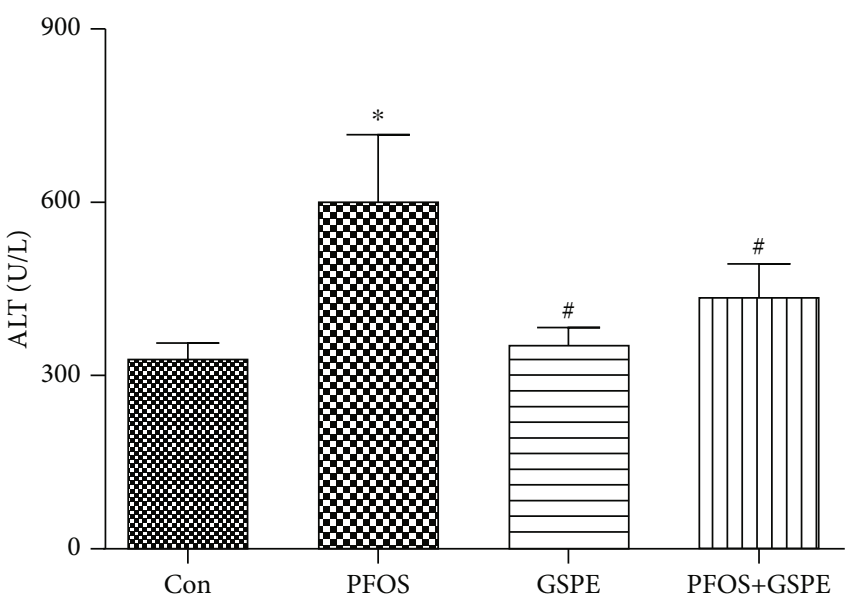

(c)

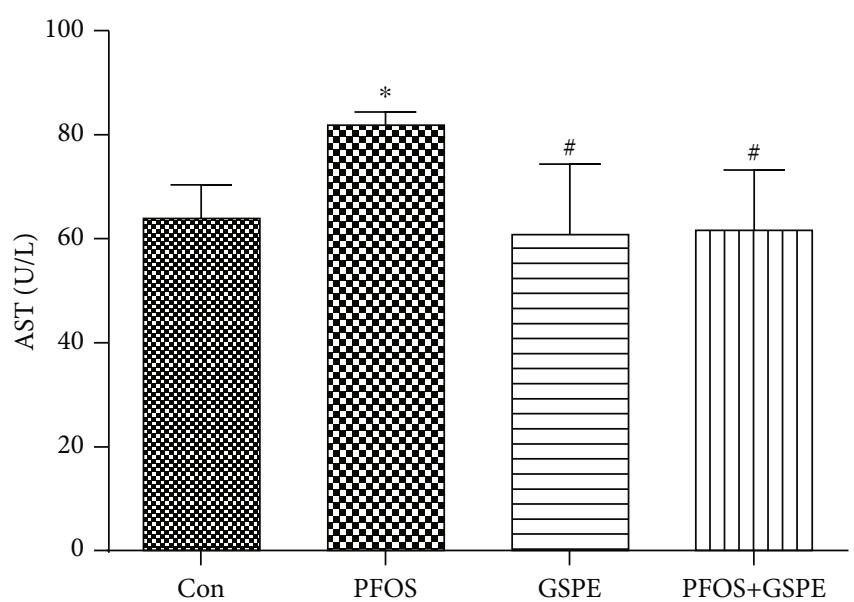

(b)

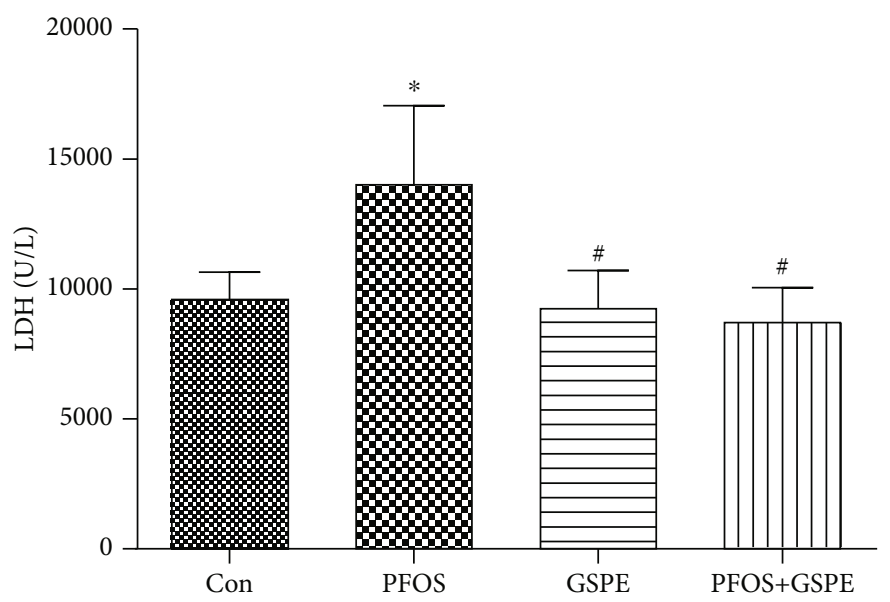

(d)

FIGURE 1: Effects of GSPE on relative liver weight and serum AST, ALT, and LDH activities in PFOS-exposed mice. Values are mean \pm SD. ${ }^{*} P<0.05$ compared to the control group; ${ }^{\#} P<0.05$ compared to the PFOS group.

remains unknown whether GSPE exerts hepatoprotection against PFOS-caused liver damage. This study hypothesizes that GSPE can attenuate PFOS-evoked steatohepatitis via improving hepatic lipid metabolism, oxidative stress, and inflammation.

\section{Materials and Methods}

2.1. Experimental Animals. Specific pathogen-free male Kunming mice weighing 20 to $22 \mathrm{~g}$ were procured from the Laboratory Animal Science Department of Jiangxi University of Traditional Chinese Medicine. The mice were housed at $25^{\circ} \mathrm{C}$ on a light/dark cycle of $12 \mathrm{~h}: 12 \mathrm{~h}$ with unrestricted diet and water intake. All procedures for animal experimentation were conducted in compliance with the guidelines of Nanchang University Laboratory Animal Research Ethics Committee (no. 20130916).

2.2. Treatments. After 1-week adaptation to the laboratory environment, the randomly selected mice were intragastrically treated once daily with PFOS $(10 \mathrm{mg} / \mathrm{kg} / \mathrm{day}, \geq 98 \%$,
Sigma-Aldrich, USA), GSPE (150 mg/kg/day, $\geq 95 \%$, Sciphar Biotechnology, China), or their combination, with 6 mice in each group. PFOS and GSPE were dissolved in distilled water. Control animals received only the equivalent volume of water. The doses of PFOS and GSPE were chosen based on the reported studies $[6,9,20,21]$. After 21 consecutive days of administration, mice were weighed and fasting blood samples were drawn under anesthesia from the retroorbital venous plexus for liver function analysis. The mice were sacrificed by cervical dislocation, and the livers were excised, weighed, and frozen in liquid nitrogen for biochemical measurement or fixed with $10 \%$ buffered formalin for histological evaluation.

2.3. Biochemical Analysis. Activities of lactate dehydrogenase (LDH), alanine transaminase (ALT), and aspartic acid transaminase (AST) in serum and contents of triglyceride (TG) and total cholesterol (TC) in homogenized livers were detected utilizing the colorimetric assay kits acquired from Jiancheng Bioengineering Institute (China). 

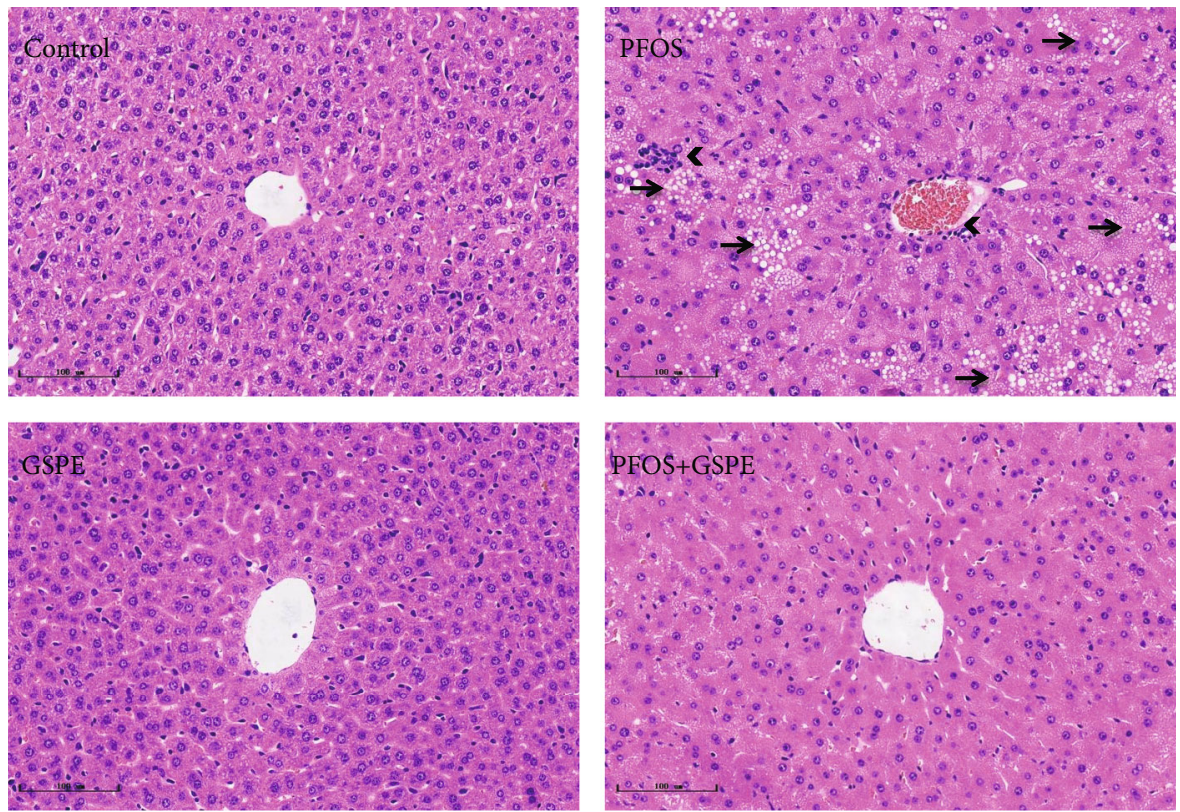

Figure 2: GSPE ameliorated PFOS-induced histological changes in the liver. Arrows indicate steatosis, and arrowheads indicate inflammatory cell infiltration. Scale bar: $100 \mu \mathrm{m}$. Magnification: 200x.

2.4. Histopathology. The formalin-fixed liver specimens were dehydrated in alcohol, infiltrated with paraffin, sliced at $5 \mu \mathrm{m}$ thickness, and stained with hematoxylin-eosin to observe hepatic morphological changes.

2.5. Real-Time Quantitative PCR. Liver RNA obtained by TRIzol was converted to cDNA with a cDNA synthesis kit purchased from TaKaRa Biotechnology (China). Quantitative SYBR Green PCR reactions were conducted using an ABI Prism 7500 apparatus. The primers used for amplification were designed as follows: CD36: $5^{\prime}$-GCCAAGCTATTGCG ACATGA- $3^{\prime}$ (forward), $5^{\prime}$-GGCATTGGCTGGAAGA ACAA-3' (reverse); FABP4: $5^{\prime}$-CTTTGTGGGAACCTGG AAGC-3' (forward), $5^{\prime}$-ATGATCATGTTGGGCTTGGC-3' (reverse); GAPDH: $5^{\prime}$-GGCAAATTCAACGGCACAGT- ${ }^{\prime}$ (forward), $5^{\prime}$-GTCTCGCTCCTGGAAGATGG-3' (reverse). The mRNA expression of CD36 and FABP4 in each sample was normalized to the corresponding GAPDH. Relative quantification was calculated using the $2^{-\Delta \Delta C T}$ method.

2.6. Oxidative Stress Assessment. Malondialdehyde (MDA) and hydrogen peroxide $\left(\mathrm{H}_{2} \mathrm{O}_{2}\right)$ generation and superoxide dismutase (SOD) activity in the liver homogenates were determined to assess hepatic lipid peroxidation, oxidant damage, and antioxidative defense using the colorimetric detection kits supplied by Jiancheng Bioengineering Institute (China).

2.7. Proinflammatory Cytokine Determination. The hepatic contents of proinflammatory cytokines interleukin-6 (IL-6) and tumor necrosis factor- $\alpha$ (TNF- $\alpha$ ) were determined using commercial ELISA test kits (Boster Biological Technology, China) following the guidance of the manufacturer.
2.8. Statistical Analysis. Experimental data were analyzed statistically by the one-way analysis of variance and Tukey's post hoc comparisons using GraphPad Prism 8.2.1 software. The results were given as means \pm SD of 4 mice. $P$ value $<0.05$ was defined as statistically significant.

\section{Results}

3.1. GSPE Lowered Liver Index and Serum Liver Enzyme Activities in Mice Exposed to PFOS. To observe the ameliorative role of GSPE in PFOS-caused hepatic injury, liver function parameters AST, ALT, and LDH were detected in mice. As illustrated in Figure 1, intragastric exposure to PFOS for 21 consecutive days resulted in a conspicuous elevation in serum AST, ALT, and LDH activities, with an increase in relative liver weight $(P<0.05)$. Nevertheless, the increased liver index and enzyme activities were resumed by combined treatment with GSPE in mice exposed to PFOS $(P<0.05)$. Treatment with GSPE alone did not influence the liver function of mice $(P>0.05)$.

3.2. GSPE Improved the Histopathologic Abnormalities in the Liver of PFOS-Exposed Mice. As displayed in Figure 2, morphological examination exhibited evident histologic features of steatohepatitis in the liver of mice administrated with PFOS, including architectural disorder, hepatocyte swelling, lipid droplet formation, and inflammatory cell infiltration. However, the pathologic abnormalities were ameliorated by supplementation of GSPE. Mice given only GSPE had the morphological structure of a normal liver.

3.3. GSPE Corrected Hepatic Lipid Metabolic Disturbance in Mice Exposed to PFOS. To further explore the therapeutic effect of GSPE on PFOS-induced steatohepatitis, levels of 


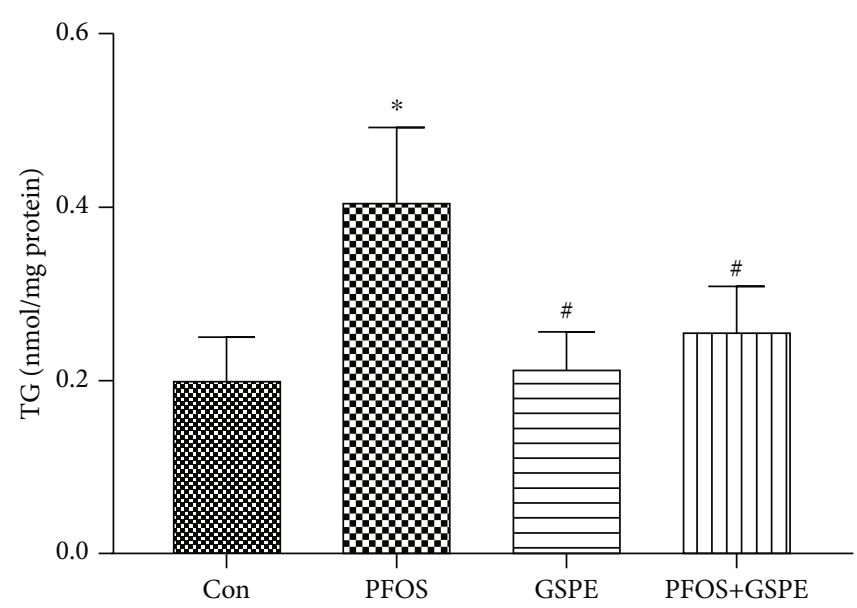

(a)

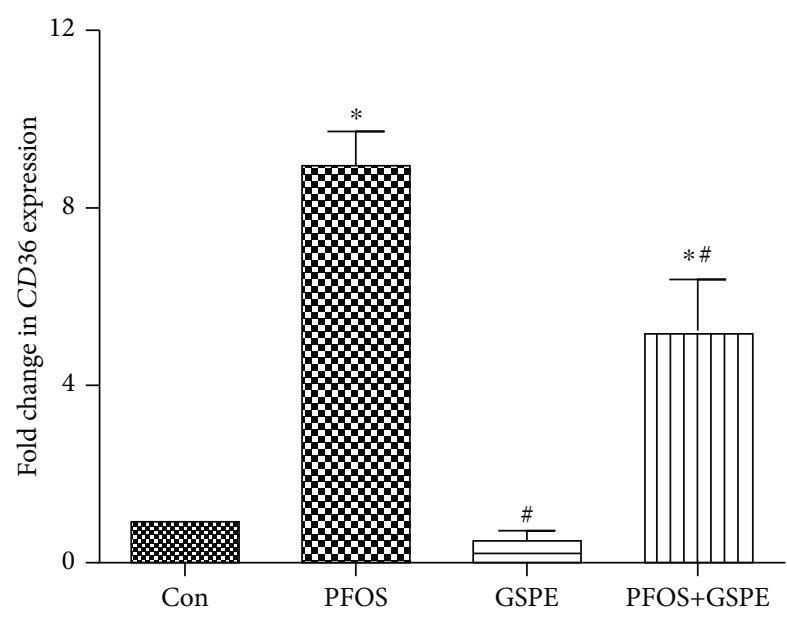

(c)

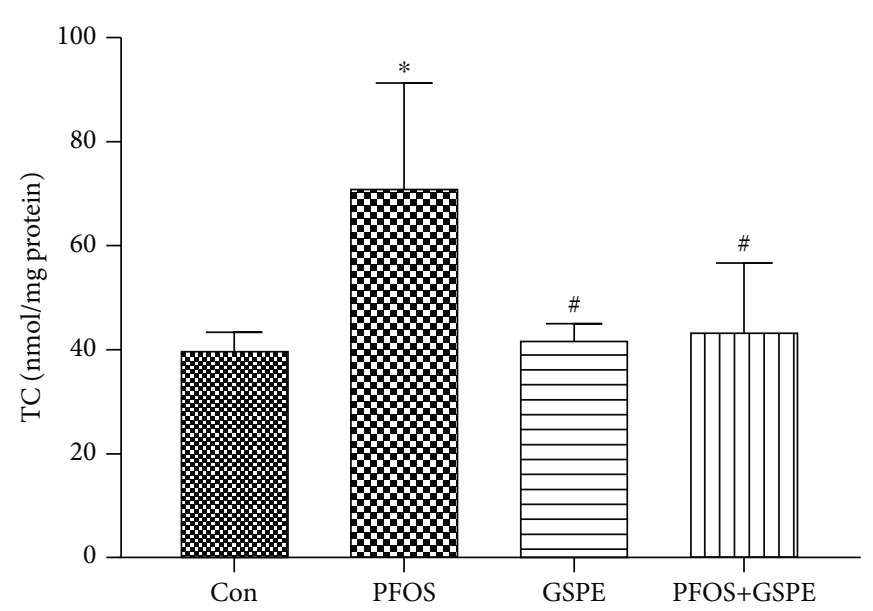

(b)

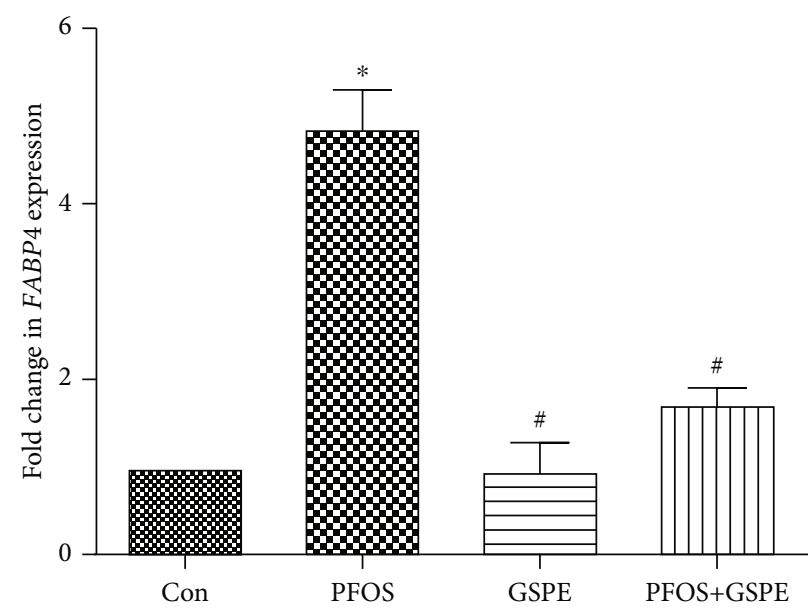

(d)

FIGURE 3: Effects of GSPE on the contents of TG and TC and mRNA expression of CD36 and FABP4 in the liver of PFOS-exposed mice. Values are mean $\pm \mathrm{SD} .{ }^{*} \mathrm{P}<0.05$ compared to the control group; ${ }^{\#} \mathrm{P}<0.05$ compared to the PFOS group.

TC and TG and expression of lipid metabolism-associated genes CD36 and FABP4 were analyzed in the liver of mice. As shown in Figure 3, the PFOS challenge induced a marked elevation in hepatic TC and TG contents $(P<0.05)$. Correspondingly, the expression of CD36 and FABP4 mRNA in the liver was notably upregulated after 21 days of PFOS exposure $(P<0.05)$. However, the increased lipid content and $C D 36$ and FABP4 expression in PFOS-exposed mice were significantly reduced by simultaneous treatment with GSPE $(P<0.05)$.

3.4. GSPE Antagonized PFOS-Initiated Oxidative Stress in the Liver. As can be seen in Figure 4, compared with the control group, the generation of oxidative stress markers MDA and $\mathrm{H}_{2} \mathrm{O}_{2}$ was enhanced and the hepatic activity of the antioxidant enzyme, SOD, was inhibited in PFOS-administrated mice $(P<0.05)$. Nonetheless, simultaneous GSPE administration reversed the increased levels of MDA and $\mathrm{H}_{2} \mathrm{O}_{2}$ and compensated for reduced SOD activity $(P<0.05)$. GSPE alone did not affect any oxidative stress indicators measured.
3.5. GSPE Attenuated PFOS-Caused Inflammation in the Liver. As presented in Figure 5, significantly increased IL-6 and TNF- $\alpha$ expressions were observed in the liver of mice exposed to PFOS compared to the control group $(P<0.05)$. Compared with the PFOS group, the increased IL- 6 and TNF- $\alpha$ were restored by combined administration with GSPE $(P<0.05)$. GSPE alone did not influence the production of these two inflammatory mediators $(P>0.05)$.

\section{Discussion}

There is increasing evidence that PFOS exerts detrimental effects on health. Considering that the liver is a main target organ for toxic insult arising from PFOS exposure, it is necessary to explore the hepatoprotection against PFOSinduced hepatic damage. Healthful nutrition intervention may be the most advisable strategy against the vulnerability to toxic chemicals. The antioxidant and anti-inflammatory properties of natural bioactive components in functional food can effectively prevent or moderate environmentally 


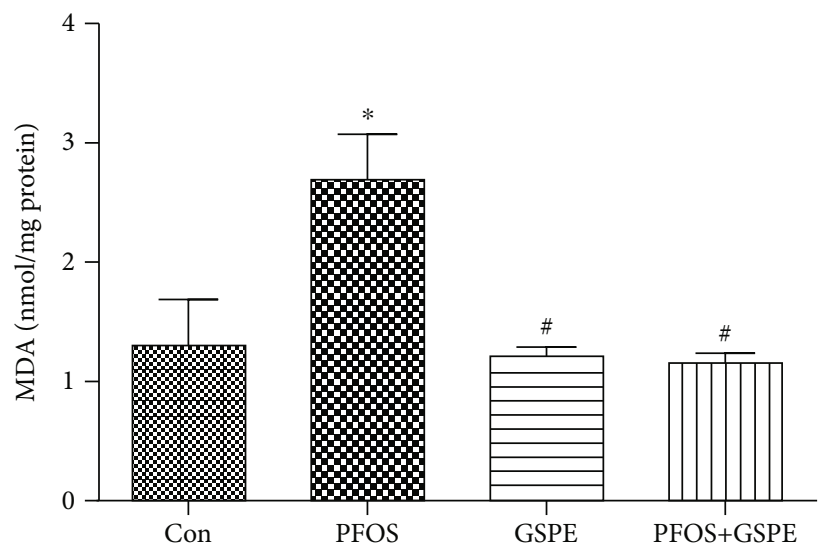

(a)

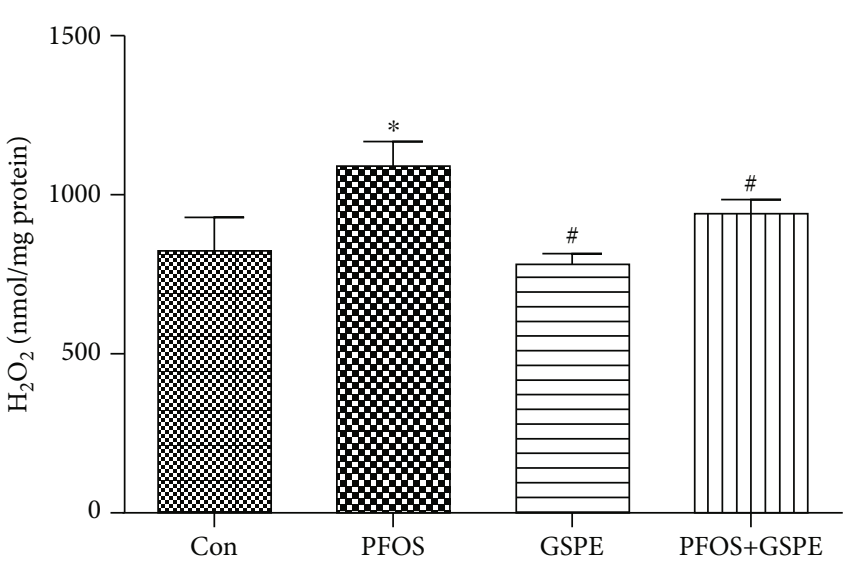

(b)

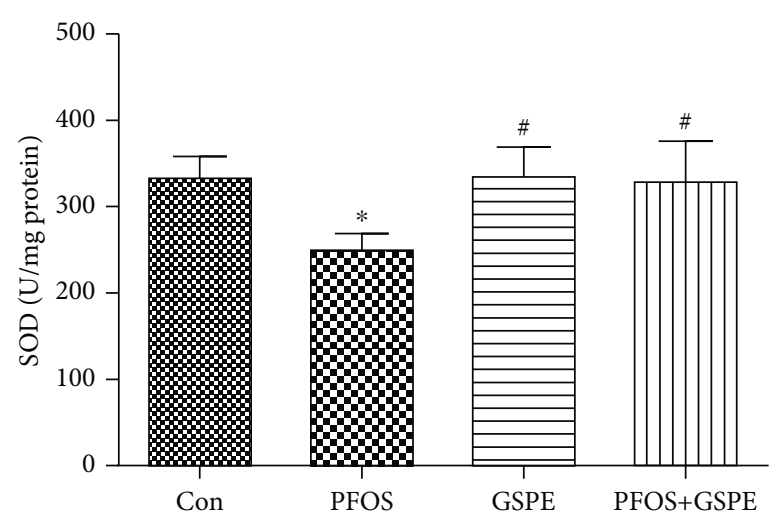

(c)

FIGURE 4: Effects of GSPE on MDA, $\mathrm{H}_{2} \mathrm{O}_{2}$, and SOD levels in the liver of PFOS-treated mice. Values are mean \pm SD. ${ }^{*} P<0.05$ compared to the control group; ${ }^{\#} P<0.05$ compared to the PFOS group.

associated diseases $[13,22]$. In the present study, mice exposed to PFOS exhibited a phenotype of nonalcoholic steatohepatitis, characterized by hepatic steatosis, hepatocyte swelling, and inflammatory infiltrate. Nevertheless, the biochemical and histological abnormalities caused by PFOS were significantly rescued by supplementation with GSPE, implying a hepatoprotective role of GSPE from PFOSevoked toxicity.

Multiple mechanisms are linked to the pathogenesis and progression of NASH [23]. It has been suggested that PFOSinduced hepatic steatosis involves the disturbance of lipid metabolism $[8,9,24,25]$. NAFLD is due to excessive fat accumulation in hepatocytes. The lipotoxicity is manifested by hepatocellular ballooning, and triacylglycerols are the major lipid component in ballooned hepatocytes. In NAFLD, increased uptake and accumulation of fatty acids propel the synthesis and storage of triglycerides in hepatocytes [26]. Undue lipid deposition in the liver can exert prooxidative and proinflammatory effects that account for the features of $\mathrm{NASH}$, and the formation of toxic metabolites during lipid metabolism is mainly responsible for the generation of ROS and induction of inflammatory mediators [27, 28].

Grape polyphenols can be proposed as a potential remedy for metabolic syndrome and NAFLD [29, 30]. It has been reported that GSPE ameliorates hepatic lipid metabolism dysfunction in diabetic mice, as well as in rats treated with a high-fat diet, $\mathrm{CCl} 4$, and lead $[17,19,31,32]$. CD36 and FABP4 are key transporters for fatty acid uptake. High hepatic levels of FABP4 have been proposed to be a predictive factor for NASH progression [33]. In NASH mice, FABP4 expression increased in the liver and overexpression of FABP4 in hepatocytes induced an elevation of proinflammatory cytokines. However, the inhibition of FABP4 significantly abrogated NASH-related inflammation [34]. CD36 directly contributes to the development and progression of fatty liver through regulating the uptake of fatty acids by hepatocytes, and the perturbation of hepatic CD36 attenuates fatty liver and associated inflammation in mice fed a high-fat diet [35]. In the present study, PFOS exposure gave rise to lipid dysmetabolism revealed by elevated contents of TG and TC and upregulated expression of lipid metabolic genes CD36 and FABP4 in the liver. However, GSPE treatment remarkably reduced lipid accumulation and CD36 and FABP4 expression in the liver of PFOS-exposed mice, suggesting that GSPE can reverse hepatic steatosis through modulating lipid metabolism.

Oxidative stress seems to be a critical factor in the pathogenesis of NASH [23]. In the process of hepatic lipid overload, free fatty acids accumulate in the liver and initiate lipid peroxidation. As a consequence of oxidative stress, lipid 


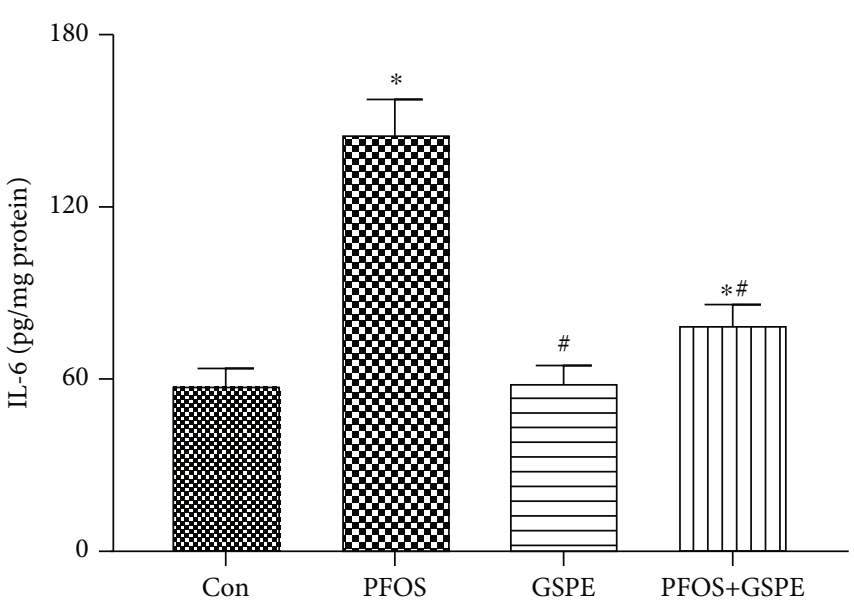

(a)

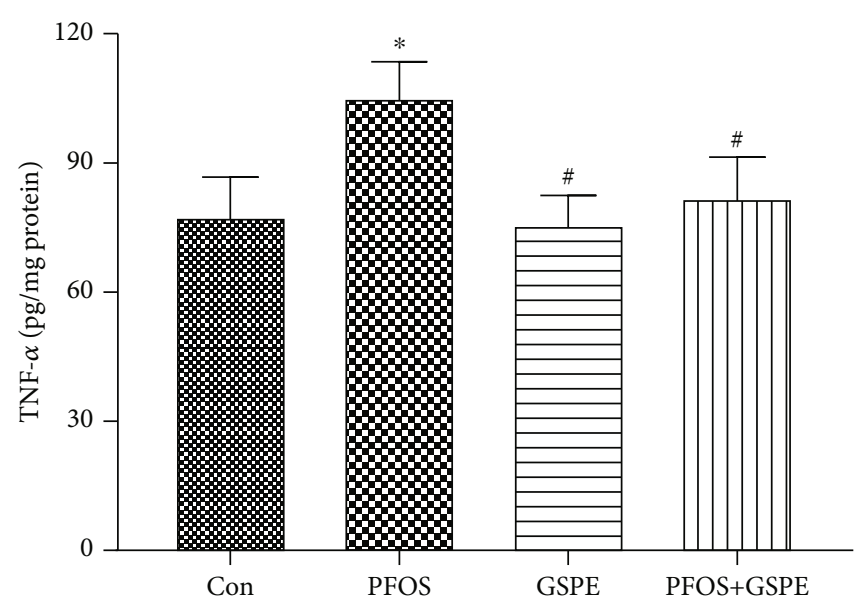

(b)

FIgURE 5: Effects of GSPE on the hepatic expression of IL- 6 and TNF- $\alpha$ in mice exposed to PFOS. Values are mean \pm SD. ${ }^{*} P<0.05$ compared to the control group; ${ }^{\#} P<0.05$ compared to the PFOS group.

peroxidation leads to severe injury in the steatotic liver [23]. In NASH patients, lipid peroxidative product MDA in erythrocytes significantly increased, and the oxidative stress in plasma was positively correlated with the severity of steatosis [36]. In this study, PFOS exposure triggered hepatic oxidative stress, which was evidenced by increased MDA generation and declined SOD activity. These results were consistent with previous findings in rodents [37-39]. Likewise, in vitro toxicity assessment demonstrated that PFOS increased ROS formation in rat hepatocytes and human HepG2 cells $[39,40]$. At present, lifestyle intervention is the main therapeutic option for $\mathrm{NASH}$, and antioxidative treatment may be an attractive candidate [23]. It has been suggested that antioxidant supplementation can ameliorate diet-induced NASH [41]. As a potent free radical scavenger and oxidative inhibitor, GSPE offers stronger protection than vitamin $\mathrm{C}$ and $\mathrm{E}$ against hepatic lipid peroxidation [42]. Studies have shown that GSPE attenuates fluoride-caused hepatotoxicity by inhibiting oxidative damage in the mouse liver and human embryo hepatocytes $[18,43]$. In our experiment, PFOSinduced hepatic oxidative stress and lipid peroxidation were dramatically alleviated by GSPE administration in mice, demonstrating a hepatoprotective potential of GSPE as a natural antioxidant in oxidative damage caused by PFOS exposure.

Inflammation is a hallmark of NASH. It is critical for the development and progression of NASH [27]. Oxidant stress is recognized to exert a pathogenic effect in steatosis-related inflammatory response [44]. Oxidative stress and consequent lipid peroxidation can activate various transcription factors, which lead to an elevation in inflammatory mediators such as TNF- $\alpha$, IL- 6 , and IL-1. These proinflammatory cytokines are of importance to recruit leukocytes into inflamed tissues $[23,45]$. Consistent with previous studies [37, 38], exposure of mice to PFOS in this study resulted in hepatic inflammation manifested by inflammatory cell infiltration, which may be linked to the augmented generation of IL- 6 and TNF- $\alpha$. Polyphenols have been proposed as adjuvant therapy for inflammation [45]. It has been shown that GSPE alleviates hepatic inflammation triggered by thioacetamide and lipopolysaccharide via upregulating the expression of proinflammatory factors [46, 47]. In the current experiment, supplementation with GSPE observably repressed the PFOSinduced overproduction of IL- 6 and TNF- $\alpha$ in hepatic tissue, indicating that GSPE exerted anti-inflammatory activity in mice exposed to PFOS.

\section{Conclusion}

GSPE supplementation can reduce TG and TC accumulation, $\mathrm{CD} 36$ and $\mathrm{FABP} 4$ expression, MDA and $\mathrm{H}_{2} \mathrm{O}_{2}$ generation, and IL- 6 and TNF- $\alpha$ production in the liver of mice exposed to PFOS, suggesting that GSPE can serve as a candidate for the prevention and therapy of PFAS-induced steatohepatitis via regulating lipid metabolism, oxidative stress, and inflammatory response. Our results provide a promising pharmacological strategy that may contribute to the protection against toxicant-associated steatohepatitis.

\section{Data Availability}

The data used to support the findings of this study are available from the corresponding author upon request.

\section{Conflicts of Interest}

The authors declare that there are no conflicts of interest.

\section{Authors' Contributions}

Tao Huang and Yurong Zhang contributed equally to this work.

\section{Acknowledgments}

This work was supported by the National Natural Science Foundation of China (81860112). 


\section{References}

[1] S. Joshibarve, I. A. Kirpich, M. C. Cave, L. Marsano, and C. J. Mcclain, "Alcoholic, nonalcoholic, and toxicant-associated steatohepatitis: mechanistic similarities and differences," Cellular and molecular gastroenterology and hepatology, vol. 1, no. 4, pp. 356-367, 2015.

[2] V. Ochoaherrera, J. A. Field, A. Lunavelasco, and R. Sierraalvarez, "Microbial toxicity and biodegradability of perfluorooctane sulfonate (PFOS) and shorter chain perfluoroalkyl and polyfluoroalkyl substances (PFASs)," Environmental Science: Processes \& Impacts, vol. 18, no. 9, pp. 12361246, 2016.

[3] J. L. Domingo, "Health risks of dietary exposure to perfluorinated compounds," Environment International, vol. 40, pp. 187-195, 2012.

[4] Y. G. Zhao, C. K. C. Wong, and M. H. Wong, "Environmental contamination, human exposure and body loadings of perfluorooctane sulfonate (PFOS), focusing on Asian countries," Chemosphere, vol. 89, no. 4, pp. 355-368, 2012.

[5] G. W. Olsen, J. M. Burris, D. J. Ehresman et al., "Half-life of serum elimination of perfluorooctanesulfonate, perfluorohexanesulfonate, and perfluorooctanoate in retired fluorochemical production workers," Environmental Health Perspectives, vol. 115, no. 9, pp. 1298-1305, 2007.

[6] B. D. Bagley, S. C. Chang, D. J. Ehresman et al., "Perfluorooctane sulfonate-induced hepatic steatosis in male SpragueDawley rats is not attenuated by dietary choline supplementation," Toxicological Sciences, vol. 160, no. 2, pp. 284-298, 2017.

[7] K. P. Das, C. R. Wood, M. T. Lin et al., "Perfluoroalkyl acidsinduced liver steatosis: effects on genes controlling lipid homeostasis," Toxicology, vol. 378, pp. 37-52, 2017.

[8] X. Liang, G. Xie, X. Wu, M. Su, and B. Yang, "Effect of prenatal PFOS exposure on liver cell function in neonatal mice," Environmental Science and Pollution Research, vol. 26, no. 18, pp. 18240-18246, 2019.

[9] H. T. Wan, Y. Zhao, X. Wei, K. Y. Hui, J. P. Giesy, and C. K. C. Wong, "PFOS-induced hepatic steatosis, the mechanistic actions on $\beta$-oxidation and lipid transport," Biochimica et Biophysica Acta, vol. 1820, no. 7, pp. 1092-1101, 2012.

[10] S. D. Geiger, J. Xiao, A. M. Ducatman, S. J. Frisbee, K. E. Innes, and A. Shankar, "The association between PFOA, PFOS and serum lipid levels in adolescents," Chemosphere, vol. 98, pp. 78-83, 2014.

[11] Y. Li, L. Barregard, Y. Xu et al., "Associations between perfluoroalkyl substances and serum lipids in a Swedish adult population with contaminated drinking water," Environmental Health, vol. 19, no. 1, p. 33, 2020.

[12] R. Jin, R. McConnell, C. Catherine et al., "Perfluoroalkyl substances and severity of nonalcoholic fatty liver in children: an untargeted metabolomics approach," Environment International, vol. 134, p. 105220, 2020.

[13] M. C. Petriello, B. J. Newsome, T. D. Dziubla, J. Z. Hilt, D. Bhattacharyya, and B. Hennig, "Modulation of persistent organic pollutant toxicity through nutritional intervention: emerging opportunities in biomedicine and environmental remediation," Science of The Total Environment, vol. 491492, pp. 11-16, 2014.

[14] H. Tapiero, K. D. Tew, G. N. Ba, and G. Mathe, "Polyphenols: do they play a role in the prevention of human pathologies?," Biomedicine \& Pharmacotherapy, vol. 56, no. 4, pp. 200-207, 2002.
[15] C. Rodriguezperez, B. Garciavillanova, E. Guerrahernandez, and V. Verardo, "Grape seeds proanthocyanidins: an overview of in vivo bioactivity in animal models," Nutrients, vol. 11, no. 10, p. 2435, 2019.

[16] D. Bagchi, S. D. Ray, D. Patel, and M. Bagchi, "Protection against drug- and chemical-induced multiorgan toxicity by a novel IH636 grape seed proanthocyanidin extract," Drugs Under Experimental and Clinical Research, vol. 27, no. 1, pp. 3-15, 2001.

[17] D. Yang, H. Jiang, J. Lu et al., "Dietary grape seed proanthocyanidin extract regulates metabolic disturbance in rat liver exposed to lead associated with PPAR $\alpha$ signaling pathway," Environmental Pollution, vol. 237, pp. 377-387, 2018.

[18] Q. Niu, P. He, S. Xu et al., "Fluoride-induced iron overload contributes to hepatic oxidative damage in mouse and the protective role of grape seed proanthocyanidin extract," Journal of Toxicological Sciences, vol. 43, no. 5, pp. 311319, 2018.

[19] N. Dai, Y. Zou, L. Zhu, H. Wang, and M. Dai, “Antioxidant properties of proanthocyanidins attenuate carbon tetrachloride (CCl4)-induced steatosis and liver injury in rats via CYP2E1 regulation," Journal of Medicinal Food, vol. 17, no. 6, pp. 663-669, 2014.

[20] W. Liu, C. Xu, X. Sun et al., "Grape seed proanthocyanidin extract protects against perfluorooctanoic acid-induced hepatotoxicity by attenuating inflammatory response, oxidative stress and apoptosis in mice," Toxicology Research, vol. 5, no. 1, pp. 224-234, 2016.

[21] M. Long, Y. Liu, Y. Cao, N. Wang, M. Dang, and J. He, "Proanthocyanidins attenuation of chronic lead-induced liver oxidative damage in Kunming mice via the Nrf2/ARE pathway," Nutrients, vol. 8, no. 10, p. 656, 2016.

[22] B. Hennig, M. C. Petriello, M. V. Gamble et al., "The role of nutrition in influencing mechanisms involved in environmentally mediated diseases," Reviews on environmental health, vol. 33, no. 1, pp. 87-97, 2018.

[23] G. H. Koek, P. R. Liedorp, and A. Bast, "The role of oxidative stress in non-alcoholic steatohepatitis," Clinica Chimica Acta, vol. 412, no. 15-16, pp. 1297-1305, 2011.

[24] L. Wang, Y. Wang, Y. Liang et al., "PFOS induced lipid metabolism disturbances in BALB/c mice through inhibition of low density lipoproteins excretion," Scientific Reports, vol. 4, no. 1, p. 4582, 2015.

[25] Z. Lv, G. Li, Y. Li et al., "Glucose and lipid homeostasis in adult rat is impaired by early-life exposure to perfluorooctane sulfonate," Environmental Toxicology, vol. 28, no. 9, pp. 532-542, 2013.

[26] S. Fiorucci, M. Biagioli, and E. Distrutti, "Future trends in the treatment of non-alcoholic steatohepatitis," Pharmacological Research, vol. 134, pp. 289-298, 2018.

[27] F. Bessone, M. V. Razori, and M. G. Roma, "Molecular pathways of nonalcoholic fatty liver disease development and progression," Cellular and Molecular Life Sciences, vol. 76, no. 1, pp. 99-128, 2019.

[28] C. Filozof, B. J. Goldstein, R. N. Williams, and A. J. Sanyal, "Non-alcoholic steatohepatitis: limited available treatment options but promising drugs in development and recent progress towards a regulatory approval pathway," Drugs, vol. 75, no. 12, pp. 1373-1392, 2015.

[29] I. Rodriguez-Ramiro, D. Vauzour, and A. M. Minihane, "Polyphenols and non-alcoholic fatty liver disease: impact and 
mechanisms," The Proceedings of the Nutrition Society, vol. 75, no. 1, pp. 47-60, 2016.

[30] M. Akaberi and H. Hosseinzadeh, "Grapes (Vitis vinifera) as a potential candidate for the therapy of the metabolic syndrome," Phytotherapy Research, vol. 30, no. 4, pp. 540-556, 2016.

[31] M. Yin, P. Zhang, F. Yu et al., "Grape seed procyanidin B2 ameliorates hepatic lipid metabolism disorders in $\mathrm{db} / \mathrm{db}$ mice," Molecular Medicine Reports, vol. 16, no. 3, pp. 2844-2850, 2017.

[32] I. Baiges, J. Palmfeldt, C. Blade, N. Gregersen, and L. Arola, "Lipogenesis is decreased by grape seed proanthocyanidins according to liver proteomics of rats fed a high fat diet," Molecular \& Cellular Proteomics, vol. 9, no. 7, pp. 1499-1513, 2010.

[33] A. Coilly, C. Desterke, C. Guettier, D. Samuel, and F. Chiappini, "FABP4 and MMP9 levels identified as predictive factors for poor prognosis in patients with nonalcoholic fatty liver using data mining approaches and gene expression analysis," Scientific Reports, vol. 9, no. 1, p. 19785, 2019.

[34] H. Jeong, J.-W. Kim, M.-S. Yang et al., "Beneficial effects of Korean red ginseng in the progression of non-alcoholic steatohepatitis via FABP4 modulation," The American Journal of Chinese Medicine, vol. 46, no. 7, pp. 1581-1607, 2018.

[35] C. G. Wilson, J. L. Tran, D. M. Erion, N. B. Vera, M. Febbraio, and E. J. Weiss, "Hepatocyte-specific disruption of CD36 attenuates fatty liver and improves insulin sensitivity in HFD-fed mice," Endocrinology, vol. 157, no. 2, pp. 570-585, 2016.

[36] C. Loguercio, V. De Girolamo, I. de Sio et al., "Non-alcoholic fatty liver disease in an area of southern Italy: main clinical, histological, and pathophysiological aspects," Journal of Hepatology, vol. 35, no. 5, pp. 568-574, 2001.

[37] Z. Lv, W. Wu, S. Ge et al., "Naringin protects against perfluorooctane sulfonate-induced liver injury by modulating NRF2 and NF- $\kappa \mathrm{B}$ in mice," International Immunopharmacology, vol. 65, pp. 140-147, 2018.

[38] R. Han, M. Hu, Q. Zhong et al., "Perfluorooctane sulphonate induces oxidative hepatic damage via mitochondriadependent and NF- $\kappa \mathrm{B} / \mathrm{TNF}-\alpha$-mediated pathway," Chemosphere, vol. 191, pp. 1056-1064, 2018.

[39] C. Wan, R. Han, L. Liu et al., "Role of miR-155 in fluorooctane sulfonate-induced oxidative hepatic damage via the Nrf2dependent pathway," Toxicology and Applied Pharmacology, vol. 295, pp. 85-93, 2016.

[40] M. R. Khansari, B. S. Yousefsani, F. Kobarfard, M. Faizi, and J. Pourahmad, "In vitro toxicity of perfluorooctane sulfonate on rat liver hepatocytes: probability of distructive binding to CYP 2E1 and involvement of cellular proteolysis," Environmental Science and Pollution Research, vol. 24, no. 29, pp. 23382-23388, 2017.

[41] A. Rezazadeh, R. Yazdanparast, and M. Molaei, "Amelioration of diet-induced nonalcoholic steatohepatitis in rats by $\mathrm{Mn}$ salen complexes via reduction of oxidative stress," Journal of Biomedical Science, vol. 19, no. 1, p. 26, 2012.

[42] D. Bagchi, A. Garg, R. L. Krohn, M. Bagchi, J. Balmoori, and S. J. Stohs, "Protective effects of grape seed proanthocyanidins and selected antioxidants against TPA-induced hepatic and brain lipid peroxidation and DNA fragmentation, and peritoneal macrophage activation in mice," General Pharmacologythe Vascular System, vol. 30, no. 5, pp. 771-776, 1998.
[43] Q. Niu, L. Mu, S. Li, S. Xu, R. Ma, and S. Guo, "Proanthocyanidin protects human embryo hepatocytes from fluorideinduced oxidative stress by regulating iron metabolism," Biological Trace Element Research, vol. 169, no. 2, pp. 174-179, 2016.

[44] Y. Ikura, M. Ohsawa, T. Suekane et al., "Localization of oxidized phosphatidylcholine in nonalcoholic fatty liver disease: impact on disease progression," Hepatology, vol. 43, no. 3, pp. 506-514, 2006.

[45] T. Hussain, B. Tan, Y. Yin, F. Blachier, M. C. B. Tossou, and N. Rahu, "Oxidative stress and inflammation: what polyphenols can do for us?," Oxidative Medicine and Cellular Longevity, vol. 2016, Article ID 7432797, 9 pages, 2017.

[46] J. Li, J. Li, S. Li et al., "Ameliorative effect of grape seed proanthocyanidin extract on thioacetamide-induced mouse hepatic fibrosis," Toxicology Letters, vol. 213, no. 3, pp. 353360, 2012.

[47] V. Pallarès, A. Fernández-Iglesias, L. Cedó et al., “Grape seed procyanidin extract reduces the endotoxic effects induced by lipopolysaccharide in rats," Free Radical Biology and Medicine, vol. 60, pp. 107-114, 2013. 